\title{
Crenças ambientais entre jovens engajados em coletivos socioambientais
}

\author{
Damaris Teixeira Paz \\ Maria Inês Gasparetto Higuchi
}

\section{RESUMO}

As crenças ambientais são construtos importantes para os estudos das relações pessoaambiente, visto que estas crenças orientam os comportamentos ecológicos. 0 objetivo deste trabalho foi analisar as crenças ambientais presentes em jovens engajados em coletivos socioambientais. Participaram do estudo 19 moças e rapazes integrantes de quatro coletivos socioambientais da região metropolitana de Manaus, Amazonas, Brasil. Os participantes responderam a um questionário com perguntas sobre o seu perfil sociodemográfico e uma escala de crenças ambientais. A partir da análise estatística, os resultados mostraram que esses jovens têm crenças predominantemente ecocêntricas e que o engajamento no grupo os ajuda a fortalecer seu próprio comportamento em relação ao cuidado ambiental e a crenças ambientais pessoais.

Palavras-chave: Engajamento socioambiental; comportamento ambiental; crenças ambientais

\section{ABSTRACT}

\section{Environmental beliefs among youngsters engaged in socioenvironmental groups}

Environmental beliefs are important constructs for the study of human-environment relations, since these beliefs guide ecological behavior. The objective of this study was to analyze the environmental beliefs present in young people engaged in social and environmental groups. Nineteen youngsters, actuating in four sócio-environmental groups, participated in the study, at the metropolitan region of Manaus. Participants answered a questionnaire with questions about their sociodemographic profile and a scale of environmental beliefs. From the statistical analysis, the results showed that these young people have predominantly ecocentric beliefs and that group engagement helps them strengthen their own behavior in relation to environmental care and personal environmental beliefs.

Keywords: Socio-environmental engagement; environmental behavior; environmental beliefs

Vários estudos em diversas perspectivas têm se dedicado a compreender o que move as pessoas a se mobilizarem, individualmente ou coletivamente, a agirem pró-ambientalmente nas mais diferentes atividades da vida cotidiana (Abrahamse, Steg, Vlek, \& Rothengatter, 2005; Chen \& Tung, 2010; Diniz, 2017; Gifford, 2006, 2008; 2011; Heath \& Gifford, 2002; Hinds \& Sparks, 2008; Paz, 2017; Raymond, Brown, \& Robinson, 2011; Stern, 2000; Swim, et al., 2011). Considerando a polissemia que orienta as mais diversas formas de pensar e agir dos humanos, não tem sido uma tarefa fácil encontrar respostas para compreender o que efetivamente aciona ações ambientalmente sustentáveis (Gifford \& Nilsson, 2014). No entanto, alguns caminhos vêm sendo revelados como indicadores possíveis de comportamento pró-ambiental. Um desses caminhos tem sido o desvelamento do tipo de crenças subjacentes ao comportamento manifestado pelas pessoas na relação com o ambiente (Bechtel, Corral-Verdugo, \& Pinheiro, 1999; Dunlap \& Van

\section{Sobre os Autores \\ D.T.P.}

orcid.org/0000-0002-9973-9811 Universidade Federal do Amazonas, (UFAM) - Manaus, AM damaris.edu.paz@gmail.com

M.I.G.H.

orcid.org/0000-0001-6525-4018 Instituto Nacional de Pesquisas da Amazônia - Manaus, AM higuchi.mig@gmail.com

\section{Direitos Autorais}

Este é um artigo aberto e pode ser reproduzido livremente, distribuído, transmitido ou modificado, por qualquer pessoa desde que usado sem fins comerciais. 0 trabalho é disponibilizado sob a licença Creative Commons CCBY-NC 
Liere, 1978; Grankvist \& Biel, 2001; Hawcroft \& Milfont, 2010; Milfont \& Duckitt, 2006).

As crenças ambientais são crenças associadas à postura humana diante das questões ambientais, as quais possibilitam explicar as ações dos humanos na relação com o ambiente e que de alguma forma podem vir a forjar comportamentos ecológicos específicos (Dunlap \& Van Liere, 1978; Pato \& Tamayo, 2005). Segundo Pato (2004), as crenças são estruturadas num sistema hierárquico, onde a pessoa associa situações tendo como referência o que é estabelecido pelo grupo social e pelo contexto cultural, além de fatores individuais (Pato, Rosa, \& Tamayo, 2005; Pato \& Tamayo, 2006, 2007). As crenças são formadas ao longo da vida das pessoas, com base no conhecimento adquirido, nas experiências vividas e mediadas pela ampla rubrica de acontecimentos, objetos e instituições presentes no contexto social onde estão inseridas (Pato \& Higuchi, 2018). Dessa forma, não há um conjunto de crenças único, mas um conjunto diferenciado entre as pessoas que refletem as características pessoais e socioculturais de cada um (Campbell-Arvai, 2015; Faver, 2013; Rosa, 2014).

Associado a esse conjunto de crenças estão incluídas inevitavelmente as informações que a pessoa tem do seu ambiente físico (Fishbein \& Ajzen, 1975), uma vez que a pessoa não vive num vácuo, e sim em relação mútua e recíproca com o entorno físico. As crenças ambientais, portanto, se constituem como uma organização das representações psicológicas acerca de uma realidade física e social (Rokeach, 1972). A partir desse sistema, os processos cognitivos e motivacionais são guiados. Dessa forma, os motivos pelos quais as pessoas cuidam (ou não) do ambiente variam de acordo com o conjunto de crenças que elas têm sobre o ambiente (Corral-Verdugo, 2001; Campbell-Arvai, 2015; Pato, 2004).

Em muitas ocasiões, no entanto, há um distanciamento entre as crenças e o comportamento. Apesar das crenças orientarem uma postura pró-ambiental, por exemplo, o comportamento não o é. Isso ocorre, principalmente, por dificuldades concretas na efetivação desse comportamento devido diversos aspectos psicossociais e contextuais. Essas dificuldades foram denominadas por Gifford (2011) como "dragões da inação". Essa incongruência entre o pensar e o agir também ocorre porque as crenças e os comportamentos, apesar de estarem fortemente relacionados, representam níveis psicológicos diferentes. As crenças representam um nível abstrato e os comportamentos um nível concreto, de modo que é mais fácil uma adaptação a um contexto pró-ambiental no nível abstrato do que no nível concreto (Bertoldo, Castro, \& Bousfield, 2013). De todo modo, mesmo ocorrendo tais fenômenos internos, percebe-se que persiste nas pessoas mais a congruência do que a incongruência entre crenças e efetiva ação.
Além desse debate das incongruências, vários estudos apontam duas polaridades que dividem o sistema de crenças: Crenças antropocêntricas (i.e., ser humano no controle da natureza) e crenças ecocêntricas (i.e., ser humano integrado à natureza; Corral-Verdugo, 2001; Campos \& Pol, 2010; Hernandez, Tabernero, \& Suárez, 2009; Rosa, Roazzi, \& Higuchi, 2015). Nesses estudos, observa-se que as pessoas com crenças mais antropocêntricas são aquelas que creem que a natureza serve para satisfazer as necessidades humanas. Já os orientados por crenças ecocêntricas creem que a natureza tem valor por si só e deve ser conservada por isso.

Nessa pesquisa, que se ocupa com jovens engajados em grupos socioambientais, as crenças teriam uma unidade reveladora de congruências com as práticas desses jovens na sociedade? Estariam mais próximas das crenças que orientam sua atuação efetiva e sua participação coletiva em atividades socioambientais? Esses questionamentos buscam revelar aspectos que possam estar presentes nas pessoas que se voltam a essas atividades de forma mais engajada.

0 engajamento em coletivos socioambientais é um comportamento de cuidado ambiental complexo, pois há uma forte doação da pessoa em prol do bem coletivo (Lee, Kim S., Kim M., \& Choi, 2014). As pessoas, principalmente os jovens, se inserem nos coletivos em busca de um espaço de participação e de aprendizado, pois em grupo, o engajamento tornase mais forte e efetivo (Carvalho, 2007; Dresner, Handelman, Braun, \& Rollwagwn-Bollens, 2015). Esses coletivos, cujos participantes desse estudo atuam, desempenham atividades de maneira voluntária com diferentes segmentos sociais nas localidades onde estão inseridos. Os grupos atuam, principalmente, na busca de uma solução para os problemas ambientais locais, além da sensibilização da sociedade para maior preservação dos recursos naturais e cuidado com o espaço físico de modo geral. Outro aspecto importante desses grupos é a dimensão social que é considerada indissociável na atuação socioambiental e na luta contra a injustiça social e desordem do ecossistema.

\section{MÉTODO}

Esse estudo de base qualitativa, descritiva-exploratória foi desenvolvido a partir de extratos de uma entrevista semiestruturada aplicada a jovens atuantes de coletivos socioambientais. Aqui configuram apenas referências sobre seu perfil sociodemográfico e respostas dadas uma escala social do tipo likert construída por Pato (2004), a Escala de Crenças Ambientais. A escala possui 26 itens com cinco graus de concordância que variam de 1 (Discordo Plenamente) a 5 (Concordo Plenamente).

Os itens da escala trazem frases que refletem crenças ambientais adequadas ao contexto brasileiro. As frases en- 
volvem diferentes dimensões dos problemas ambientais encontrados no país e no mundo como os resíduos, o uso dos recursos naturais e poluição das indústrias, por exemplo. Os itens da escala configuram crenças antropocêntricas (10 itens) e crenças ecocêntricas (16 itens).

Os coletivos foram identificados a partir de um levantamento inicial numa etapa da Conferência de Juventude e Meio Ambiente em Manaus, ocorrida em 2015, e pela própria indicação dos demais entrevistados de coletivos dentro do perfil da pesquisa. Os participantes foram selecionados pelo critério de acessibilidade, com participação no grupo há pelo menos 6 meses. A aplicação do questionário se deu após prévio acordo com os entrevistados de data, local e hora e teve duração média de 15 minutos. 0 estudo foi devidamente aprovado pelos critérios éticos previstos na resolução 466/2012. Sendo aprovado pelo Comitê de Ética em Pesquisa com Seres Humanos da Universidade Federal do Amazonas sob parecer de No 1.660.619.

Participaram da pesquisa 19 jovens $(8 \mathrm{~F}, 11 \mathrm{M})$, todos participantes de quatro coletivos socioambientais da região metropolitana de Manaus-AM. Esses jovens tinham idade de 16 a 29 anos (média 22,4; dp 3,8) e estavam engajados no grupo em média de 3,7 anos (dp 2,7). A grande maioria dos jovens $(89,5 \%)$ já havia concluído ou estava cursando o ensino superior, alguns em nível de pós-graduação (15,8\%), embora a formação superior desses jovens não estivesse diretamente ligada às áreas de conhecimento ambiental. A maioria $(89,5 \%)$ revelou estar empenhada em trabalhar com questões socioambientais no seu cotidiano pessoal e profissional. Apenas dois participantes, mais jovens, ainda cursando o ensino médio, relataram não fazer essa transversalidade em suas atividades.

Todos os participantes desenvolvem alguma atividade além das grupais, $47,4 \%$ deles só estudam, outros $47,4 \%$ estudam e trabalham e um jovem $(5,2 \%)$ apenas trabalha. Entre eles, há quem more sozinho ou com o cônjuge (31,6\%), mas a maioria ainda mora com a família $(68,4 \%)$. Nenhum deles relata ter filhos. Considerando a renda familiar per capita, os jovens participantes podem ser considerados de classe média (Associação Brasileira de Empresas de Pesquisa [ABEP], 2015), pois 17 participantes declararam ter uma renda per capita de $\mathrm{R} \$ 667,00$ a $\mathrm{R} \$ 2.000,00$, e dois afirmaram possuir renda familiar per capita acima de $\mathrm{R} \$ 3.000,00$, sendo, portanto, considerado de classe média alta. Tal renda no estilo de vida urbano brasileiro permite a esses jovens ter acesso mínimo a recursos para o lazer, informação, formação profissional e cultura.

Pouco mais da metade dos participantes $(52,6 \%)$, confirma já ter participado de outros coletivos como grupos de jovens de igrejas ou outros movimentos tais como jovens punk, contra a corrupção, movimento estudantil (centros acadêmicos), ONGs, projetos acadêmicos na área ambiental e projetos sociais. Alguns deles ainda participam de outros coletivos como os já citados, e até outras organizações na área ambiental. Isso mostra que o engajamento cívico observado nesses jovens é expressivo.

Os dados foram analisados a partir de análise estatística descritiva, com verificação das médias de respostas para os itens da escala e de algumas informações sobre os jovens.

\section{RESULTADOS}

Ao analisar a escala de crenças ambientais, verificou-se que jovens obtiveram uma média geral de 3,3. Considerando a possibilidade de 1 a 5 essa média indica uma predominância de posicionamentos de concordância com as afirmativas contidas na escala. Entretanto, pelo fato da escala ter dois construtos diferenciados, a análise das crenças vistas separadamente permite um cenário revelador. Ao separar os constructos que evidenciam respectivamente crenças ecocêntricas e antropocêntricas, observou-se que as médias mais altas evidenciaram que esses jovens têm predominantemente crenças ambientais ecocêntricas. A média obtida dos graus de concordância foram substancialmente maiores nos itens que expressam crenças ecocêntricas $(m=4,18)$ e menores nos itens de crenças antropocêntricas $(m=1,84)$. Nas Figuras 1 e 2 se observa as crenças ecocêntricas e antropocêntricas e a distribuição das respectivas médias.

Figura 1. Crenças ecocêntricas

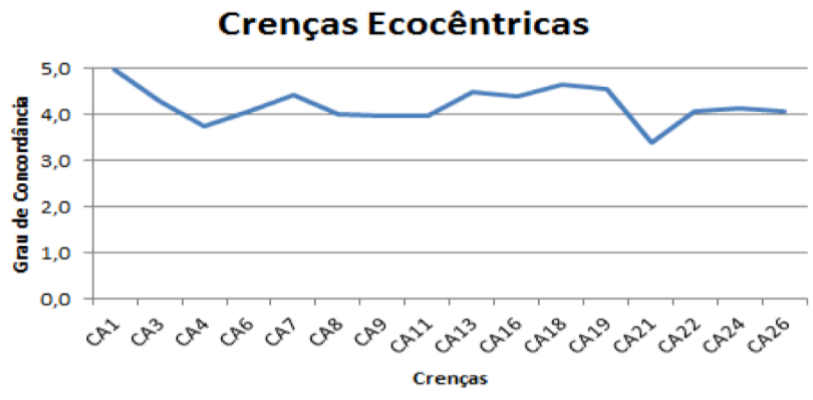

Nota: Figura 1. Média obtida pelos jovens nos itens de crenças ecocêntricas. CA1 - Evitar desperdícios dos recursos naturais deve ser um compromisso de todos nós brasileiros; CA3 - A reciclagem contribui para a diminuição dos problemas ambientais gerados pelo uso abusivo de papéis; CA4 - Reciclar latas de alumínio é uma fonte de economia para as indústrias; CA6 - A luta dos ambientalistas ajuda a melhorar a nossa qualidade de vida; CA7 - Se as coisas continuarem como estão, vivenciaremos em breve uma catástrofe Ecológica; CA8 Evitar a compra de produtos poluentes faz com que as empresas se preocupem mais com o meio ambiente; CA9 - As pessoas deveriam boicotar as empresas que poluem o meio ambiente para exigir produtos ecologicamente corretos; CA11 - Se existissem mais campanhas 
esclarecendo a população sobre os problemas ambientais, a situação brasileira estaria melhor; CA13 - Alimentos produzidos organicamente são melhores para a saúde humana; CA16 - O homem é o responsável pelo desequilíbrio na natureza; CA18 - É possível manter o equilíbrio ecológico e ter uma boa qualidade de vida; CA19 - Os homens estão abusando do meio ambiente; CA21 - Os problemas ambientais são consequência da vida moderna; CA22 - A interferência dos seres humanos na natureza frequentemente produz consequências desastrosas; CA24 - Separar o lixo conforme o tipo ajuda na preservação do meio ambiente; CA26 - 0 consumismo agrava os problemas ambientais.

Figura 2. Crenças antropocêntricas

\section{Crenças Antropocêntricas}

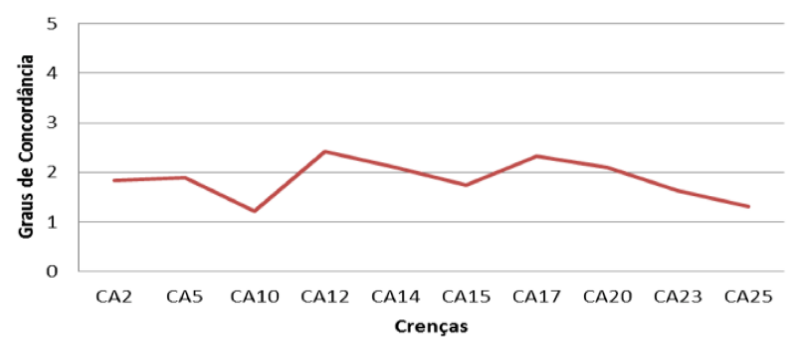

Nota: Médias obtidas pelos jovens nos itens de crenças antropocêntricas. CA2 - As pessoas exageram os problemas ambientais provocados pelo uso do automóvel; CA5 - O Brasil é um país com muitas riquezas naturais e é impossível que essas riquezas acabem apenas pelas ações humanas; CA10 - 0 lixo é responsabilidade apenas do órgão de limpeza urbana; CA12 - O governo deveria se preocupar mais com os problemas sociais do que com os ambientais; CA14 - Os ecologistas estão preocupados demais com as plantas e os animais, e se esquecem das pessoas; CA15 -A natureza tem uma capacidade inesgotável de se recuperar dos danos provocados pelas ações humanas; CA17 - Os recursos naturais estão aí para servir ao homem; CA20 - A nossa qualidade de vida depende diretamente dos bens de consumo que possuímos; CA23 - 0 equilíbrio da natureza é forte o suficiente para se ajustar aos impactos das nações industriais modernas; CA25 - Usar muito papel causa problemas sérios, mas eu não posso fazer nada sobre isso.

Em análises suplementares foram encontradas leves diferenças entre as médias das respostas de acordo com o gênero (Antropocêntricas: F: 1,71; M: 1,96) (Ecocêntricas: F: 4,25; M: 4,13). Tal resultado indica que os homens expressaram, em média, graus de concordância relativamente menores do que as mulheres nas crenças ecocêntricas e relativamente maiores nas crenças antropocêntricas. Essas diferenças, no entanto, não se configuram significativas sob o ponto de vista estatístico. Não foram observadas outras tendências em relação à idade ou o tempo de participação no grupo e predominância de crenças ambientais.

\section{DISCUSSÃo}

Esses jovens participantes dessa pesquisa se diferenciam da maior parte dos jovens brasileiros, pois segundo Silva, Macedo e Figueiredo (2015), ao analisarem o perfil dos jovens trabalhadores brasileiros, a partir dos dados da Pesquisa Nacional por Amostragem de Domicílios (PNAD) de 2006 e de 2013 , verificaram que os jovens de 18 a 24 (maioria dos jovens aqui pesquisados) trabalham em média 36,51 horas semanais e possuem em média 9,8 anos de estudo. 0 que significa que em 2013, a maior parte dos jovens trabalhadores não tinha se formado no ensino médio (equivalente a 12 anos de estudo). Ainda sobre a formação, apesar do acesso ao ensino superior ter aumentado no país, os índices ainda são baixos. Segundo Corbucci (2016), no Censo de 2010 apenas $18,7 \%$ dos jovens de 18 a 24 anos haviam tido esse acesso. Os jovens aqui entrevistados fazem parte dessa população que teve acesso ao ensino superior, seja em instituições de ensino públicas ou particulares.

A alta escolaridade desses jovens se mostra um fator relevante no envolvimento deles em coletivos socioambientais e na predisposição de se engajar socioambientalmente, como afirmam outras pesquisas desenvolvidas nessa área (Pisano \& Hidalgo, 2014; Côrtes, Dias, Fernandes, \& Pamplona, 2016). Engajamento esse que se fortalece na inserção e convívio com outras pessoas que atuam nesses coletivos. Tais aspectos foram verificados no estudo de Diniz e Pinheiro (2014) e Karpudewan, Ismail e Ruth (2012), os quais verificaram que o conhecimento sobre as questões ambientais contribui para a construção de crenças ambientais mais voltadas ao cuidado do entorno e dos recursos naturais.

A inserção deles num grupo também é um fator de contribuição para fortalecimento de suas próprias crenças e refinamento de seu comportamento pró-ambiental, uma vez que a ação dos pares retroalimenta os dispositivos atitudinais de seus integrantes. No caso específico das crenças ambientais, todo o conjunto de crenças é construído a partir do contexto social e cultural, fruto das experiências diretas e indiretas das pessoas (Fishbeim \& Ajzen, 1975; Rosa, 2014).

Esses resultados mostram que as crenças ecocêntricas prevalecem entre os jovens participantes de coletivos socioambientais. Na literatura as crenças são vistas como variáveis disposicionais que indicam uma predisposição a determinado comportamento, no caso aqui analisado indica maior predisposição ao cuidado ambiental (Rosa, 2014). Tais crenças salientam que a natureza tem valor intrínseco a si e que o ser humano está integrado ao ambiente. Isso demonstra que as razões para o comportamento pró-ambiental desses jovens está também no fato deles acreditarem que é necessário cuidar do ambiente. Fator este, que pode estar diretamente relacionado com a participação deles em coletivos socioambientais (Côrtes \& Moretti, 2013; Kapuderwan et al., 2012; 
Campos \& Pol, 2010), pois os grupos não só se tornam um espaço para suas crenças, como também as fortalecem em função da atuação coletiva de proteção ambiental. Ao evidenciarem crenças predominantemente ecocêntricas que os movem, esses jovens acreditam na interdependência dos seres humanos e do ambiente e na necessidade da proteção ambiental. Esse estudo corrobora com o pressuposto de que as crenças ecocêntricas auxiliam na construção do comportamento pró-ambiental, neste caso, em especial na mobilização socioambiental em coletivos. As crenças ecocêntricas podem ainda ter sido fortalecidas a partir do envolvimento dos jovens com o grupo. Considerando o tempo médio de participação dos jovens em coletivos socioambientais que é bastante elevado diante das suas idades. Sendo tal participação, portanto uma experiência de grande relevância nas suas vidas.

Ficou evidente neste estudo que os jovens que buscaram um grupo para se engajar socioambientalmente possuem crenças que embasam comportamentos pró-ambientais. Crenças essas que ajudam a estruturar as suas atitudes diante das questões socioambientais, contribuindo para a formação da sua identidade ambiental (Dresner et al., 2015; CarvaIho, n.d.).

\section{CONSIDERAÇÕES FINAIS}

Como as crenças são formadas a partir das experiências diretas e indiretas das pessoas, e considerando que os jovens estão engajados no mínimo há seis meses nos coletivos, infere-se que a formação das crenças ecocêntricas recebe contribuições da participação dos jovens nos coletivos. Então, as experiências obtidas nas atividades de cuidado ambiental e o próprio convívio com outras pessoas engajadas socioambientalmente fortalece esse tipo de crenças. Além disso, colabora para a formação de uma identidade ambiental, a daqueles jovens que se preocupam e se mobilizam para reagir a situações de depredação ambiental.

O estudo aqui proposto corrobora com estudos já desenvolvidos de que as crenças, por serem orientadoras do comportamento, têm um papel importante nas mudanças necessárias para a construção de sociedades mais sustentáveis. 0 engajamento socioambiental é importante e tem sido fortalecido nesses coletivos. Destaca-se, portanto, que a participação dos jovens nesses coletivos pode ser relevante para a formação de cidadãos sustentáveis, e que o incentivo na existência desses coletivos pode ser um fator de especial distinção em busca da formação de comportamentos próambientais.

É importante destacar, no entanto, as limitações desse estudo que não aborda fatores da vida pessoal e familiar desses jovens que também possam ter contribuído com a cons- trução das crenças ambientais. Além disso, novas pesquisas para verificar se tais crenças estão também presentes entre jovens que não estejam envolvidos em grupos socioambientais. Considera-se que tais estudos possam vir a elucidar outros aspectos da formação dessas crenças e do comportamento pró-ambiental.

\section{FINANCIAMENTO}

Para a realização desta pesquisa recebemos financiamento em forma de bolsa de estudos da Fundação de Amparo à Pesquisa do Amazonas e do Conselho Nacional de Desenvolvimento Científico e Tecnológico.

\section{DECLARAÇÃO DA CONTRIBUIÇÃO DOS AUTORES}

Certificamos que todos os autores participaram suficientemente do trabalho para tornar pública sua responsabilidade pelo conteúdo. A contribuição de cada autor pode ser atribuída como se segue:

DTP: conceitualização, investigação, metodologia, redação, tabulação de dados, validação, visualização, análise formal dos dados.

MIGH: conceitualização, metodologia, redação, validação, visualização, análise formal dos dados.

\section{REFERÊNCIAS}

Associação Brasileira de Empresas de Pesquisa [ABEP]. (2015). Critério de Classificação Econômica Brasil. Recuperado de www.abep.org

Abrahamse, W., Steg, L., Vlek, C., \& Rothengatter, T. (2005). A review of intervention studies aimed at household energy conservation. Journal of Environmental Psychology, 25, 273-291. https://doi.org/10.1016/j.jenvp.2005.08.002.

Bechtel, R., Corral-Verdugo, V. \& Pinheiro, J. (1999). Environmental beliefs U.S., Brazil and Mexico. Journal of Cross-cultural Psychology, 30, 122-128. http://dx.doi.org/10.1177/0022022199030001008903 0001008

Bertoldo, R., Castro, P., \& Bousfield, A.B. (2013). Proenvironmental beliefs and behaviours: two levels of response to environmental social norms. Revista Latinoamericana de Psicología, 45 (3), 437-448. https://doi.org/10.14349/rlp.v45i3.1485 
Campbell-Arvai, V. (2015). Food-related environmental beliefs and behaviours among university undergraduates: A mixed-methods study. International Journal of Sustainability in Higher Education. 16 (3), 279-295, https://doi.org/10.1108/IJSHE-06-2013-0071

Campos, C.B., \& Pol, E. (2010). As crenças ambientais de trabalhadores provenientes de empresa certificada por SGA podem predizer comportamentos pró-ambientais fora da empresa? Estudos de Psicologia, 15(2), 199206.

https://doi.org/10.1590/S1413$294 \times 2010000200009$

Carvalho, I. C. M. (2007). Subjetividade e sujeito ecológico: contribuições da psicologia social para a educação ambiental. Em A. F. S. Guerra \& J. E. Taglieber (Orgs.), Educação ambiental: Fundamentos, práticas e desafios (1a Ed., Vol Único, pp. 29-36). Itajai (SC): Editora da UNIVALI,.

Carvalho, I. C. M. (n.d.). Sujeito ecológico: A dimensão subjetiva da ecologia.. Recuperado de http://www.dominiopublico.gov.br/download/texto/m e4655.pdf.

Chen, M. F., \& Tung, P. J. (2010). The moderating effect of perceived lack of facilities on consumers' recycling intentions. Environment and Behavior, 42, 824-844. https://doi.org/10.1177/0013916509352833

Corbucci, P. R. (2016). Desigualdades no acesso dos jovens brasileiros à educação superior. Em, E. R. A. Silva \& R. U. Botelho, (Eds), Dimensões da experiência juvenil brasileira e novos desafios às políticas públicas. Brasília: IPEA.

Corral-Verdugo, V. (2001). Comportamiento proambiental: Una introducción al estudio de las conductas protectoras del ambiente. Santa Cruz de Tenerife, Espanha: Resma.

Côrtes, P. L., \& Moretti, S. L. A. (2013). Consumo verde: Um estudo transcultural sobre crenças, preocupações e atitudes ambientais. Revista Brasileira de Marketing, 12(3), 45-76. doi: 10.5585/remark.v12i3.2592

Côrtes, P. L., Dias, A. G., Fernandes, M. E. S. T., \& Pamplona, J. M. V. (2016). Comportamento Ambiental: Estudo Comparativo entre Estudantes Brasileiros e Portugueses. Ambiente e Sociedade, 19(3), 113-134. http://dx.doi.org/10.1590/1809-

4422ASOC139099V1932016
Diniz. L.R. (2017). Identidade e engajamento político de ativistas do movimento ambientalista do Norte e Nordeste do Brasil. Tese (Doutorado em Psicologia). Universidade Federal de Pernambuco.

Diniz, R. F., \& Pinheiro, J. Q. (2014). Cuidado ambiental em tempos de sustentabilidade: Relação de compromisso pró-ecológico e orientação de futuro. PSICO, 45(3), 387-394. http://dx.doi.org/10.15448/19808623.2014.3.17321

Dresner, M., Handelman, C., Braun, S., \& Rollwagwn-Bollens, G. (2015). Environmental identity, pro-environmental behaviors, and civic engagement of volunteer stewards in Portland area parks. Environmental Education $\begin{array}{llll}\text { Research, } & 21 & \text { (7), }\end{array}$ https://doi.org/10.1080/13504622.2014.964188

Dunlap, R. E., \& Van Liere, K. D. (1978). The "New Environmental Paradigm": A proposed measuring instrument and preliminary results. Journal of Environmental Education, 9, 10-19. https://doi.org/10.1080/00958964.1978.10801875

Faver, C. (2013). Environmental beliefs and concern about animal welfare: Exploring the connections. Journal of Sociology \& Social Welfare, 04(XL), 149-168. Recuperado http://scholarworks.wmich.edu/jssw/vol40/iss4/9

Fishbein, M., \& Ajzen, I. (1975). Belief, attitude, intention, and behavior: An introduction to theory and research. Reading, MA: Addison-Wesley.

Gifford, R., \& Nilsson, A. (2014). Personal and social factors that influence pro-environmental concern and behaviour: A review. International Journal of Psychology, 3(49), 141157..https://doi.org/10.1002/ijop.12034

Gifford, R. (2006). A general model of social dilemmas. International Journal of Ecological Economics and Statistics, 5, 23-40.

Gifford, R. (2008). Toward a comprehensive model of social dilemmas. In A. Biel, D. Eek, T. Gärling, \& $M$. Gustafsson (Eds.), New issues and paradigms in research on social dilemmas. New York, NY: Springer. 
Gifford, R. (2011). The dragons of inaction: Psychological barriers that limit climate change mitigation and adaptation. American Psychologist, 66, 290-302. https://doi.org/10.1037/a0023566

Grankvist, G., \& Biel, A. (2001). The importance of beliefs and purchase criteria in the choice of eco-labeled food products. Journal of Environmental Psychology, 21, 405-410. https://doi.org/10.1006/jevp.2001.0234

Hawcroft, L. J., \& Milfont. T. L. (2010). The use (and abuse) of the new environmental paradigm scale over the last 30 years: A meta-analysis. Journal of Environmental Psychology, 30 143-158. https://doi.org/10.1016/j.jenvp.2009.10.003

Heath,Y., \& Gifford, R. (2002). Extending the theory of planned behavior: Predicting the use of public transportation. Journal of Applied Social Psychology, 32, 2154-2189. https://doi.org/10.1111/j.1559-1816.2002.tb02068.x

Hernández, B., Tabernero, C., \& Suárez, E. (2009). Psychosocial motivations and self-regulation processes that activate environmentally responsible behavior. In J. Valentin, \& L. Gamez (Eds.) Environmental Psychology: New Developments. Nova Science Publishers.

Hinds, J., \& Sparks, P. (2008). Engaging with the natural environment: The role of affective connection and identity. Journal of Environmental Psychology, 28, 109120. https://doi.org/10.1016/j.jenvp.2007.11.001

Karpudewan, M., Ismail, Z., \& Roth, W. M. (2012). Promoting pro-environmental attitudes and reported behaviors of Malasian pre-service teachers using green chemistry experiments. Environmental Education Research, 18(3), 375-389.

https://doi.org/10.1080/13504622.2011.622841

Lee, Y., Kim, S., Kim, M., \& Choi, J. (2014).Antecedents and interrelations of three types of pro-environmental behavior. Journal of Business Research, 67, 2097-2105. https://doi.org/10.1016/j.jbusres.2014.04.018.

Milfont, T. L., \& Duckitt, J. (2006). Preservation and utilization: Understanding the structure of environmental attitudes. Medio Ambiente y Comportamiento Humano, 7(1), 29-50.
Pato, C. M. L. (2004). Comportamento ecológico: Relações com valores pessoais e crenças ambientais. Tese (Doutorado em Psicologia). Universidade de Brasília, Brasília.

Pato, C., \& Tamayo, A. (2006). Valores, creencias ambientales y comportamiento ecológico de activismo. Medio Ambiente y Comportamiento Humano, 17 (1), 51-66.

Pato, C., \& Tamayo, A. (2007). Valores, creencias ambientales y comportamiento ecológico de ahorro de agua y energía. Revista de Psicología Social, 22, 245-253. https://doi.org/10.1174/021347407782194407

Pato, C., Rosa, M., \& Tamayo, A. (2005). Creencias y comportamiento ecológico: Un estudio empírico con estudiantes brasileños. Medio Ambiente $y$ Comportamiento Humano, 6 (1), 5-22.

Pato, C., \& Higuchi (2018). Crenças ambientais e atitudes ecológicas. Em S. Cavalcante \& G. A. Elali (Eds.) Psicologia Ambiental: Conceitos para a leitura da relação pessoa-ambiente. Petrópolis: Vozes. Manuscrito submetido à publicação.

Paz, D. T. (2017). Aspectos constitutivos do engajamento e participação de jovens em coletivos socioambientais na região metropolitana de Manaus/AM. Dissertação (Mestrado em Ciências do Ambiente e Sustentabilidade na Amazônia). Manaus: UFAM.

Pisano, I., \& Hidalgo, M. C. (2014). Testing the cross-national social bases of environmentalism: A current and comparative analysis of conservation a behaviors. Psico. 45 (3), 395-405.

Raymond, C. M., Brown, G., \& Robinson, G. M. (2011). The influence of place attachment, and moral and normative concerns on the conservation of native vegetation: A test of two behavioural models. Journal of Environmental Psychology, 31, 323-335. https://doi.org/10.1016/j.jenvp.2011.08.006

Rokeach, M. (1972). Beliefs, attitudes and values. A Theory of Organization and Change, London: Jossey-Bass. 
Rosa, D. C. C. B. (2014). Teorias sobre a floresta e funções de apego: Um estudo sobre a relação das pessoas com a Amazônia. Tese de Doutorado, Pós-Graduação em Psicologia Cognitiva, Universidade Federal de Pernambuco, Recife. Recuperado de http://repositorio.ufpe.br/handle/123456789/10934

Rosa, D. C. C. B., Roazzi, A., \& Higuchi, M. I. G. (2015). PSICAMB - Perfil de afinidade ecológica: Um estudo sobre os indicadores da postura perante a natureza. PSICO, 46(1), 139-149. https://doi.org/10.15448/19808623.2015.1.17415

Silva, E. R., Macedo, D. M. B., \& Figueiredo, M. M. A. (2015). Conciliação dos estudos, trabalho e vida familiar na juventude brasileira. Organização Internacional do Trabalho (OIT); OIT Escritório no Brasil; Instituto de Pesquisa Econômica Aplicada (IPEA). Brasília: OIT.
Stern, P. C. (2000). Toward a coherent theory of environmentally significant behavior. Journal of Social Issues, 56, 407-424. doi:10.1111/0022-4537.00175

Swim, J. K., Stern, P. C., Doherty, T., Clayton, S., Reser, J. P., Weber, E. U., Gifford, R., Howard, G. S. (2011). Psychology's contributions to understanding and addressing global climate change mitigation and adaptation. American Psychologist, 66, 241-250. https://doi.org/10.1037/a0023220

Recebido em: 14/09/2017 Primeira decisão editorial em: 20/12/2017 Aceito em: 14/03/2017 